\title{
Heated Wind Particle's Behavioural STUDY BY THE CONTINUOUS WAVELET TRANSFORM AS WELL AS THE FRACTAL ANALYSIS
}

\author{
Sabyasachi Mukhopadhyay ${ }^{1}$, Sanmoy Mandal $^{2}$, P.K.Panigrahi ${ }^{3}$, Asish \\ Mitra $^{4}$ \\ 1,2,3 Physical Sciences Department at IISER, Kolkata \\ ${ }^{1}$ sabyasachi.unique@gmail. com \\ ${ }^{4}$ College of Engineering \& Management, Kolaghat \\ mitra_asish@yahoo.com
}

\section{ABSTRACT}

Nowadays Continuous Wavelet Transform (CWT) as well as Fractal analysis is generally used for the Signal and Image processing application purpose. Our current work extends the field of application in case of CWT as well as Fractal analysis by applying it in case of the agitated wind particle's behavioral study. In this current work in case of the agitated wind particle, we have mathematically showed that the wind particle's movement exhibits the "Uncorrelated" characteristics during the convectional flow of it. It is also demonstrated here by the Continuous Wavelet Transform (CWT) as well as the Fractal analysis with matlab 7.12 version.

\section{KEYWORDS}

Continuous Wavelet Transform (CWT), Fractal Analysis, White Noise.

\section{INTRODUCTION}

For the characterization of the Wind speed and Wind energy, the role of mathematical tools is inevitable. We know that the Weibull distribution is an important mathematical tool especially for reliability and maintainability analysis. The suitable values for both shape parameter and scale parameters of Weibull distribution are important for selecting locations of installing wind turbine generators [2]. For the analysis of low wind speed, the utility of Discrete Hilbert Transform was first introduced by the authors [2]. Actually the Hilbert transformation, similarly used as Hilbert transformer which deals with signals in time domain, was first introduced to signal theory by Denis Gabor [3]. Today it plays a significant role in signal processing. The theory on this has been introduced by Hahn [4] in detail. Till now, more researches have focused on the parameters for the probability density distribution. As an alternative, the output from a DHT filter is quite possible to be applied in Wind engineering. Mukhopadhyay et al. showed that Discrete Hilbert Transform (DHT) can be used as minimum phase type filter for characterizing and forecasting purpose of Wind speed data [6]. Already Panigrahi et al. showed the utility of Wavelet in weather

Rupak Bhattacharyya et al. (Eds) : ACER 2013, pp. 169-174, 2013. (C) CS \& IT-CSCP 2013

DOI : $10.5121 /$ csit.2013.3216 
related application [1]. After getting success with DHT in case of Wind Energy study, the concept of 'Wavelet Transform' and 'Factuality' has been applied in case of Wind data analysis under summer weather in this current work. The similar application was done in case of a particle pointer within a heated liquid [7].

\section{THEORY}

\subsection{Continuous Wavelet Transform(CWT)}

The wavelet transform of a continuous time signal, $x(t)$, is defined as: $T(a, b)=\frac{1}{\sqrt{a}} \int_{-\infty}^{\infty} x(t) \psi^{*}\left(\frac{t-b}{a}\right) d t$, where $\psi^{*}(t)$ is the complex conjugate of the wavelet function of $\psi(t), a$ is the dilation parameter of the wavelet and $b$ is the location parameter of the wavelet. In order to be classified as a wavelet, the function must satisfy certain mathematical criteria. These are:

A wavelet must have finite energy: $E=\int_{-\infty}^{\infty}|\psi(t)|^{2} d t<\infty$.

If $\psi(f)$ is the Fourier transform of $\psi(t)$,

$$
\psi(\omega)=\int_{-\infty}^{\infty} \psi(t) e^{-j w t} d t
$$

Then the following condition must hold:

$$
C_{g}=\int_{0}^{\infty} \frac{|d \omega|^{2}}{\omega} d \omega<\infty
$$

This implies that the wavelet has no zero frequency component, i.e., $\psi(0)=0$, or to put it another way, it must have a zero mean. Equation (1) is known as the 'Admissibility Condition' and $C_{g}$ is called 'Admissible Constant'. The value of $C_{g}$ depends on the chosen wavelet. For complex (or analytic) wavelets, the Fourier transform must both be real and vanish for negative frequencies. The contribution to the signal energy at the specific $a$ scale and $b$ location is given by the two-dimensional wavelet energy density function known as the 'Scalogram':

$$
E(a, b)=|T(a, b)|^{2} .
$$

The total energy in the signal may be found from its wavelet transform as follows:

$$
E=\frac{1}{C_{g}} \int_{-\infty}^{\infty} \int_{0}^{\infty} \frac{1}{a^{2}}|T(a, b)|^{2} d a d b
$$

In practice a fine discretisation of the continuous wavelet transform is computed where usually the $b$ location is discretised at the sampling interval and the $a$ scale is discretised logarithmically. The $a$ scale discretisation is often taken as integer powers of 2; however, we 
use a finer resolution in our method where the $a$ scale discretisation is in fractional powers of two. This discretisation of the continuous wavelet transform (CWT) is made distinct from the discrete wavelet transform (DWT) in the literature. In its basic form, the DWT employs a dyadic grid (integer power of two scaling in $a$ and $b$ ) and orthonormal wavelet basis functions and exhibits zero redundancy. Our method, i.e. using a high resolution in wavelet space as described above, allows individual maxima to be followed accurately across scales, something that is often very difficult with discrete orthogonal or dyadic stationary wavelet transforms incorporating integer power of two scale discretisation.

\subsection{Fractal Pattern}

Fractal pattern is a geometrical pattern having self-affinity in every scale. Example- White Noise, Monofractal, Multifractal. In fractal analysis, the most important parameter is the "Hurst Exponent ${ }^{e}$. It can be expressed as below: $H \propto$ Variance(S), where $\mathrm{H}$ is the Hurst Exponent and $\mathrm{S}$ is the number of points. $\mathrm{H}<0.5$, Uncorrelated, under White Gaussian Noise condition. $0.5<\mathrm{H}$ $<1$, Correlated.

\subsection{White Noise}

A continuous time random process $w(t)$ where $t \in R$ a white noise process if and only if its mean function and autocorrelation function satisfy the following: $\mu_{w}(t)=E\{w(t)\}=0$. $R_{w w}\left(t_{1}, t_{2}\right)=E\left\{w\left(t_{1}\right) w\left(t_{2}\right)\right\}=\left(N_{0} / 2\right) \delta\left(t_{1}-t_{2}\right)$.i.e., it is a zero mean process for all time and has infinite power at zero time shifts since its autocorrelation function is the Dirac delta function.

The above autocorrelation function implies the following power spectral density: $S_{w w}(w)=\frac{N_{0}}{2}$, since the Fourier transform of the delta function is equal to 1 . Since this power spectral density is the same at all frequencies, we call it white as an analogy to the frequency spectrum of white light. A generalization to random elements on infinite dimensional spaces, such as random fields, is the white noise measure.

\section{MeThOdolOGY}

Here at first the uncorrelated behavior of wind particle is shown mathematically. Thereafter the result is verified with the Continuous Wavelet Transform (CWT) as well as with the Fractal analysis.

\section{Mathematical Modelling}

We know from Ref [9] that under the white noise condition the Co-variance function of any time series signal $x(t)$ is $K_{\omega}(\tau)=\delta(\tau)$. So, $K_{\omega}(t-\tau)=\delta(t-\tau)$, where $\tau$ is a time delay. Now, from the definition of the auto-correlation we have 


$$
\int_{t=0}^{\infty} K_{\omega}(t) K_{\omega}(t-\tau) d t=\int_{t=0}^{\infty} \delta(t) \delta(t-\tau) d t=[\delta(\tau)]^{2}
$$

Now, we all know that for $\tau \neq 0, \delta(\tau)=0$. So, in that case it is clear that the under white noise condition time series of signals are un-correlated. Here, for our work purpose we consider the time series of Wind data.

\section{RESULTS \& DisCUSSIONS}

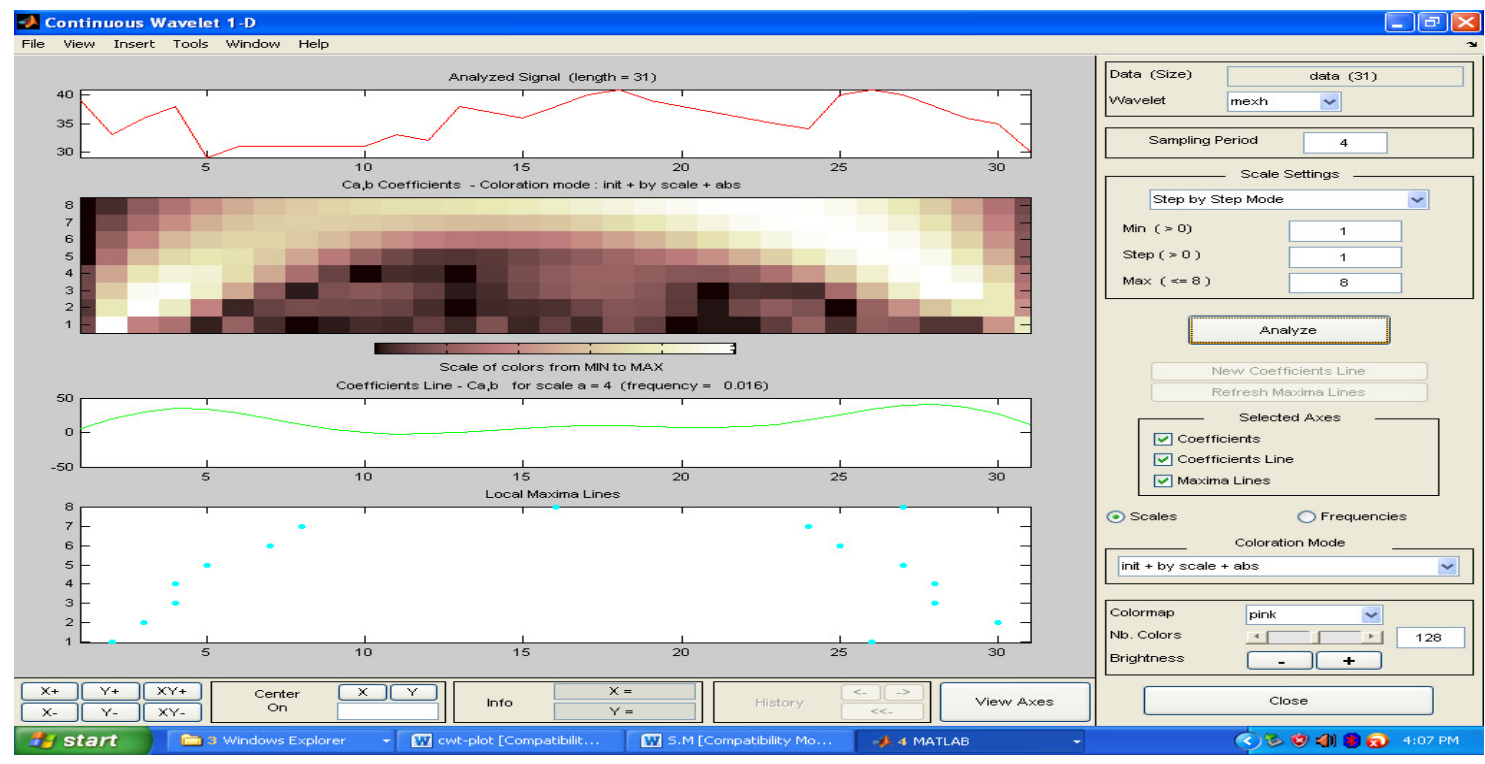

Fig-1 Continous Wavelet Transform (CWT) plot for Wind data

The $1^{\text {st }}$ section plot shows the wind speed data graph. The next section plot shows the testing of the Self-Similarity by correlation between the Wavelet Coefficients. Here the response plot is not Self-similar i.e., uncorrelated (White Gaussian noise characteristics). The $3^{\text {rd }}$ section shows the Wavelet Coefficient plot. The last section plot shows the Local Maxima which shows the agitated wind samples throughout the month of May, 2012.

After analyzing with the CWT, the wind speed dates are analyzed next with the Fractal analysis. 


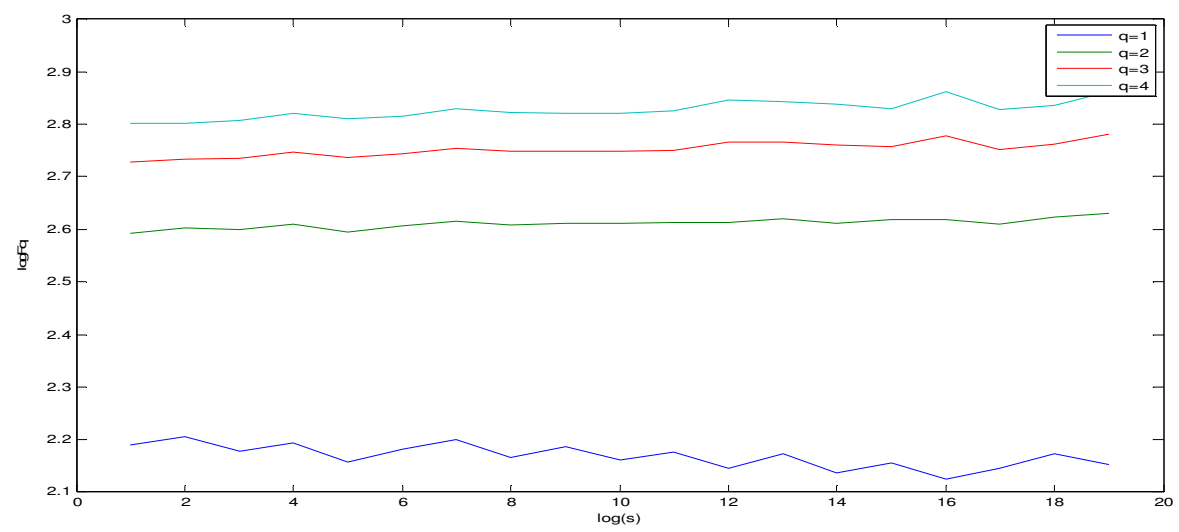

Fig $-2 \log (\mathrm{s})$ vs. $\log \mathrm{Fq}$

Here $\log (\mathrm{s})$ vs. $\log \mathrm{Fq}$ plot is taken with respect to $\mathrm{q}=2$. Here, $\mathrm{s}=$ scale and $\log \mathrm{Fq}=$ Logarithm of the scaling function $\mathrm{F}(\mathrm{s}, \mathrm{q})$.

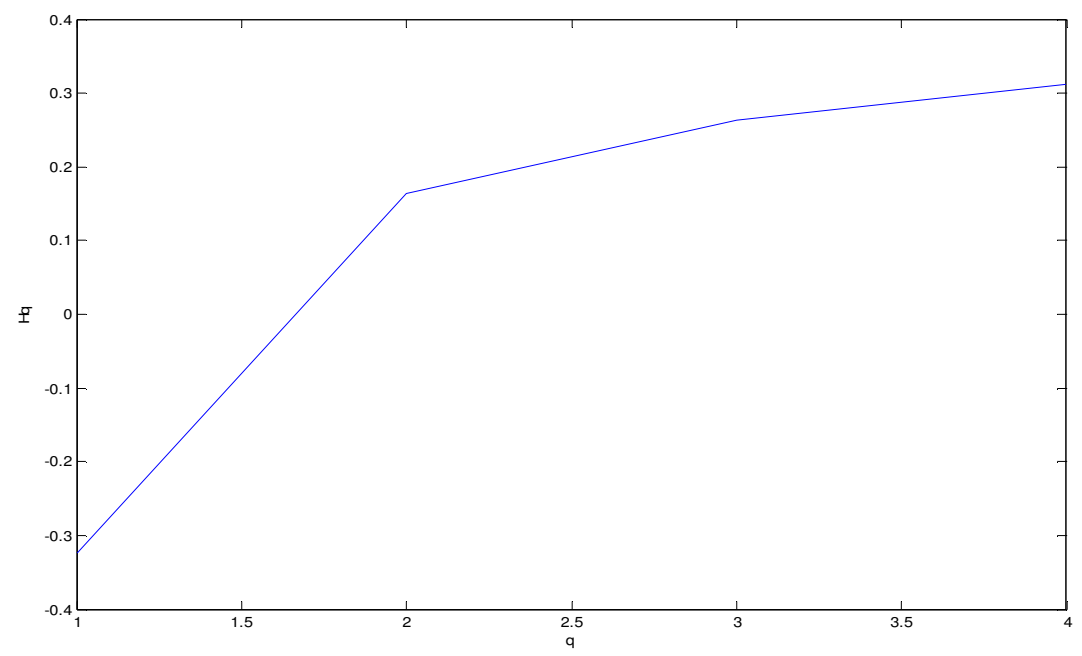

Fig-3 q th order Hurst Exponent (Hq) plot

From this plot it is clear that here value of the Hurst Exponent of q-th order $(\mathrm{Hq})$ is lies under 0.5 i.e., exhibiting White Noise characteristics.

\section{Conclusions}

From the above plots and the mathematical modeling it is clear that the wind particle shows the uncorrelated behavior under White Noise condition which is shown by CWT as well as by the Fractal Analysis in this work. The authors hope that this work may guide researchers to move forward in this field. 


\section{REFERENCES}

[1] Panigrahi, P.K., Maniraman, P., Lakshmi, P.A., Yadav, R.R., 2006, Correlations and periodicities in Himalayan tree ring widths and temperature anomalies through wavelets, http://arxiv.org/abs/nlin/0604002v1.

[2] Bhattacharya, P., Mukhopadhyay, S., 2011, Weibull Distribution for Estimating the Parameters and Application of Hilbert Transform in case of a Low Wind Speed at Kolaghat, The International Journal of Multiphysics, Volume-5, No-3.

[3] Gabor, D.,1946, Theory of communications, J. Inst. Electr. Eng. Part III 93, 429.

[4] Hahn, S.L., 1996, Hilbert Transforms in Signal Processing, Artech House, Inc., Boston, London.

[5] Proakis,2007, Digital Signal Processing, Pearson Education India, ISBN 8131710009, 9788131710005.

[6] Mukhopadhyay, S., Bhattacharya, P., Bhattacharjee, R., Bose, P.K., 2012, Discrete Hilbert Transform as Minimum Phase Type Filter for the Forecasting and the characterization of Wind Speed, CODIS International IEEE Conference Proceeding, Kolkata, India.

[7] Mukhopadhyay, S., Mitra, A., 2012, "Observation of Spectral Density \& Autocorrelation Characteristics for White Gaussian Noise by a Particle Pointer within a Heated Liquid”, ICFMSA 2012, Calcutta Mathematical Society, Kolkata, India.

[8] Chonavel, T., 2005, Statistical Signal processing, Springer International Edition, ISBN- 81-8128379-1, page-59.

[9] Barkat, M., Signal Detection and Estimation, Artech House.

\section{AUTHORS}

Sabyasachi Mukhopadhyay: Mr.Sabyasachi Mukhopadhyay completed B.Tech in Electronics \& Communication Engg. from College of Engineering \& Management, Kolaghat in July,2012. Currently he is pursuing his research work as Project Assistant in Physical Sciences department of IISER, Kolkata. Till now he has 16 numbers of International Journals, International/ National Conference Proceedings with winning the best research paper award once. His areas of research interests are Digital Signal \& Image Processing, Renewable Energy and Graph Theory.

Sanmoy Mandal: Mr. Sanmoy Mandal is currently pursuing third year Integrated BS-MS in Indian Institute of Science Education Research, Kolkata. Interested in Mathematical Physics, Quantum Information processing and Non-standard Formulation of Quantum Mechanics.

Prof. (Dr.)Prasanta K. Panigrahi: Currently Prof.(Dr.)Panigrahi is the chairman of the Physical Sciences Department of IISER, Kolkata. He completed his PhD from University of Rochester, New York, USA. He also had Post Doctorial research work from University of Illinois at Chicago, USA. He delivered invited talks in eminent universities of India and Overseas. He is the elected Fellow of the Gujarat Academy of Science, India. He is Referee of Physical Review Letters, Physical Review B, Journal of Physics, Pattern Recognition Letters, Physics Letter A, Pramana, European Physics Letters. Till now he has more than 800 publications in reputed Journals and Conference Proceedings. His areas of research interest are BoseEinstein Condensates, Cold fermions, Nonlinear Dynamics, Quantum Computation and Quantum Information, Non-Commutative Field Theory and Many body physics, Wavelet Transform.

Dr. Asish Mitra: Currently Asst. Prof. in the Department of Physics of "College of Engineering and Management, Kolaghat", West Bengal, India. He completed his PhD from Jadavpur University, Kolkata. He also had Post Doctorial research work from the same university. He is Reviewer of the International Journal: Heat and Mass Transfer, Publisher: Springer Berlin/Heidelberg and d WSEAS. He has several publications in reputed Journals and Conference Proceedings. His area of research interest is Computational Fluid Dynamics. 